\title{
A miotomia do cricofaríngeo: artigo de revisão
}

\section{Cricopharyngeal myotomy: review article}

\author{
Maria Camila Castilho do Amaral ${ }^{1}$, Ana Maria Furkim ${ }^{2}$
}

\begin{abstract}
RESUMO
A miotomia do cricofaríngeo é um procedimento cirúrgico que pode ser indicado para pacientes com disfagia orofaríngea que, em geral, caracterize-se por distúrbio motor limitado à fase faríngea da deglutição, incoordenação cricofaríngea ou relaxamento incompleto do Esfíncter Esofágico Superior (EES). A indicação da miotomia, porém, tem sido discutida e aplicada em muitos casos, sem efeito na melhora da dinâmica da deglutição. O objetivo deste estudo foi verificar os critérios de indicação e eficácia da miotomia do cricofaríngeo, com base nos estudos da fisiologia da deglutição em pacientes com disfagia orofaríngea. Realizou-se, então, uma revisão da literatura sobre este procedimento e a descrição de seus resultados na dinâmica da deglutição. Os resultados demonstraram que os melhores indicadores para uma miotomia bem sucedida são: fase oral eficiente e boa elevação laríngea durante a deglutição. Assim, para uma adequada indicação da miotomia do cricofaríngeo, não se deve avaliar apenas a fase faríngea e o funcionamento isolado do EES, mas a coordenação e relação entre as fases oral e faríngea da deglutição.
\end{abstract}

DESCRITORES: Músculos faríngeos/cirurgia; Cartilagem cricóide/cirurgia; Esfíncter esofágico superior/fisiologia; Músculos faringeos/fisiologia

\section{INTRODUÇÃO}

A deglutição é um processo dinâmico e complexo que requer a contração e relaxamento coordenado de muitos grupos musculares, que agem seqüencialmente para propelir o bolo alimentar da cavidade oral para o estômago ${ }^{(1)}$. Durante o trânsito do alimento pelo tubo digestivo tal seqüência de ações deve ser sincrônica, pois cada ação vai determinar a eficiência da ação seguinte. Um bom controle oral do bolo alimentar facilita o envio adequado do bolo à câmara faríngea ${ }^{(1-3)}$.

Após a anamnese, avaliação clínica e a realização de exames complementares, os médicos e terapeutas que acompanham o paciente disfágico vão prognosticar e planejar os procedimentos visando a sua reabilitação. Dentre as possibilidades de tratamentos para reabilitação estão as terapias fonoaudiológicas, terapias nutricionais, além de intervenções mais invasivas e cirúrgicas ${ }^{(3-7)}$.

(1) Especialista em Motricidade Oral - Disfagia em Âmbito Hospitalar pelo Centro de Especialização em Fonoaudiologia Clínica - CEFAC - São Paulo (SP), Brasil; Especializanda em Motricidade Orofacial pelo Centro de Especialização em Fonoaudiologia Clínica - CEFAC - São Paulo (SP), Brasil. (2) Doutora em Fonoaudiologia pela Universidade Federal de São Paulo UNIFESP - São Paulo (SP), Brasil; Diretora do Instituto de Gerenciamento em Fonoaudiologia e Deglutição - IGD - São Paulo (SP), Brasil; Professora do Centro de Especialização em Fonoaudiologia Clínica - CEFAC - São Paulo (SP), Brasil.

Endereço para correspondência: Maria Camila Castilho do Amaral. R. Matias Aires, 268/64, Consolação, São Paulo - SP, CEP 01309-020. E-mail: mcamilacamaral@yahoo.com.br

Recebido em: 30/6/2006; Aceito em: 12/3/2007
A miotomia do cricofaríngeo é um procedimento cirúrgico, que consiste em cortar as fibras do músculo cricofaríngeo ou EES, com a intenção de liberar ou melhorar a passagem do bolo alimentar pela transição faringoesofágica $(\mathrm{TFE})^{(8-12)}$.

Os aspectos fisiopatológicos dos distúrbios da deglutição mais correlacionados com indicação de miotomia do cricofaríngeo foram: distúrbio motor limitado à fase faríngea da deglutição, incoordenação da sequiência de contração céfalo-caudal da musculatura cricofaríngea, relaxamento incompleto do esfíncter esofágico superior (EES) ou incoordenação na abertura do EES durante a passagem do bolo ${ }^{(7-8,10,13-21)}$.

Tem sido relatado que, em pacientes com disfagia neurológica, um melhor trânsito faringoesofágico pode ser observado quando a deglutição voluntária ainda se mostra intacta, ou seja, a fase oral permanece eficiente. A propulsão pobre da língua atrasa o reflexo de início da deglutição faríngea e reduz a peristalse faríngea, o que justifica as pobres chances de melhora nesses pacientes apesar da miotomia. Contudo, em pacientes com elevação intacta da laringe, a miotomia cricofaríngea pode aumentar a abertura do $\operatorname{EES}^{(5,9-10,13,19,22)}$.

Muitas miotomias, entretanto, têm sido indicadas levando em consideração apenas o aspecto resíduo em faringe e/ ou acima do EES. Se a relação de interdependência entre as fases e estágios é fundamental para a eficiência da deglutição, a fase oral auxilia com sua eficiência a abertura da transição faringo-esofágica. Esses primeiros aspectos não podem ser os únicos a serem considerados - a despeito dos achados da 
fase oral e dos achados da relação entre a fase oral e faríngea da deglutição ${ }^{(6-7,18-19,22-24)}$.

O objetivo deste estudo foi verificar os critérios de indicação e eficácia da miotomia do cricofaríngeo, com base nos estudos da fisiologia da deglutição, em pacientes com disfagia orofaríngea. Realizou-se, então, uma revisão da literatura sobre este procedimento e a descrição de seus resultados na dinâmica da deglutição.

\section{REVISÃO DA LITERATURA}

A deglutição é um processo fisiológico complexo que ocorre por meio de respostas coordenadas, seqüenciais, simultâneas e inter-relacionadas que asseguram o transporte eficaz do bolo alimentar e a proteção do trato respiratório enquanto os alimentos são engolidos e digeridos ${ }^{(1)}$.

Devido a sua origem embriológica comum, partilhando um mesmo caminho na faringe, durante a respiração, o esfíncter velofaríngeo e a laringe estão abertos e o esfíncter superior do esôfago fica fechado nesse momento, evitando a entrada de ar no estômago. Quando a deglutição se inicia, a faringe assume a função de via digestiva, fechando-se a via aérea para a proteção contra a aspiração de bolo alimentar ou saliva ${ }^{(1)}$.

A deglutição envolve o córtex, o tronco cerebral, muitos músculos e seis nervos cranianos: trigêmio ( $\mathrm{V}$ par) facial (VII), glossofaríngeo (IX par), vago (X par), acessório (XI par) e hipoglosso (XII par) e é dividido em três fases: oral, faríngea e esofágica ${ }^{(1-2)}$.

A fase oral, voluntária, é a fase de preparo em que ocorre a captação e o processamento do alimento por meio da mastigação (trituração, pulverização e ensalivação) e formação de bolo alimentar, qualificando-o, posicionando-o sobre a língua e ejetando-o para a faringe $\mathrm{e}^{(1)}$.

Em seguida, ocorre o início da fase faríngea da deglutição, que se dá com o impacto do alimento sobre os receptores sensoriais, táteis e de pressão, localizados no palato mole, na orofaringe, nos arcos fauciais e na porção faríngea da língua que ativa o complexo motor, sendo propelido para a faringe ${ }^{(3,4)}$.

O bolo é direcionado pela contração tônica dos músculos, com movimentos peristálticos dos constritores, estreitando o lúmen faríngeo seqüencialmente, separando a faringe em compartimentos a partir das mudanças nos gradientes de pressão. Esta fase dura aproximadamente um segundo ${ }^{(3,4)}$.

Depois do início da fase faríngea, não há mais pausa, ocorrendo quatro eventos seqüenciais estereotipados: contração da faringe, fechamento velofaringeano, elevação e fechamento da laringe e abertura do cricofaríngeo ou segmento faringoesofágico ${ }^{(23)}$.

O fechamento faringoesofágico se dá pela função ativa da parte cricofaríngea do músculo constritor inferior. É um vedamento estável com pressão intraluminal aumentada, exceto durante a deglutição, a eructação ou o vômito ${ }^{(3)}$. As funções de esfíncter e de alta pressão regional não se devem ao estado de contração mantida do músculo cricofaríngeo por suas características anatômicas e por ser de tipo estriado esquelético, mas sim pela contração da musculatura faríngea, resultado da ação de pinça exercida entre a coluna cervical (C5/C6) e a laringe (cartilagem cricóide) no nível da transição faringoesofágica $(\mathrm{TFE})^{(5-10)}$.

O esfíncter esofágico superior (EES), músculo cricofaríngeo, constituído pelo complexo constritor inferior da faringe, quando em repouso, permanece fechado, o que impede a entrada de ar no esôfago. Com a constrição faríngea, o esfíncter se relaxa, abrindo-se para a passagem do bolo e fechando-se $\operatorname{logo}$ em seguida ${ }^{(5,8-10)}$. Estudos realizados com manometria combinada e videofluoroscopia demonstram que a passagem do bolo alimentar da faringe ao esôfago depende do movimento de pistão da língua e do diferencial de pressão entre a orofaringe e o esôfago ${ }^{(8,11,23)}$.

O bolo é levado para o estômago a partir dos movimentos peristálticos do esôfago ${ }^{(25)}$. O esfíncter esofágico inferior, localizado na porção caudal do esôfago, se fecha para impedir o refluxo do conteúdo estomacal ${ }^{(26)}$. Assim se constitui a terceira fase da deglutição, durante aproximadamente cinco segundos, sendo esta involuntária e independente da idade do indivíduo ${ }^{(3,24)}$.

A videomanometria oferece um método de controle da posição do plano de monitoramento, o que permite uma distinção precisa entre a pressão interna do bolo e uma pressão contrátil, importante no entendimento da fisiologia da deglutição ${ }^{(4)}$. Exames de manometria demonstraram que o valor do aumento da pressão variou nos diferentes níveis dentro da faringe, estando mais alto no nível do segmento esôfago faríngeo, menor no nível do constritor inferior da faringe, menor no cricofaríngeo e menor ainda no nível da base da língua ${ }^{(4,27)}$.

A atividade do constritor faríngeo é um dos principais componentes da deglutição faríngea, impulsionando o bolo para o esôfago. Quando esta atividade é prejudicada, o material de bolo retido na faringe pode penetrar na laringe e traquéia $^{(4)}$.

Devido à abertura do cricofaríngeo é que ocorre o diferencial de pressão entre a orofaringe e o esôfago. Os mecanismos que levam à abertura do cricofaríngeo são: relaxamento do EES, tração pela elevação do conjunto hióide-laringe e encurtamento da faringe, forças de pressão do bolo iniciadas pelo movimento de propulsão da língua e baixa complacência do $\operatorname{EES}^{(23,24)}$.

O esfíncter esofágico superior (EES) ou transição faringoesofágica (TFE), com 2 a $4 \mathrm{~cm}$ de extensão, é uma zona de alta resistência, registrando-se pressão positiva média que varia de 18 a $145 \mathrm{mmHg}$, durante o repouso, através do método manométrico ${ }^{(4,8,10)}$.

O fascículo cricofaríngeo do constritor inferior da faringe não pode ser considerado o único responsável por esta zona de alta pressão, pois a alta pressão registrada manometricamente para a transição faringoesofágica (TFE) excede em extensão o limite anatômico do músculo cricofaríngeo, de inserção antero-lateral, a cada lado, nas bordas postero-laterais da cartilagem cricóide, não configurando assim um anel muscular completo ${ }^{(8,10,24)}$.

Por isto, o músculo cricofaríngeo, do tipo estriado, não se mantém contraído por muito tempo, só se relaxa durante o esforço da deglutição. Participam associados a ele, uma 
parte do fascículo tireofaríngeo acima e a musculatura esofágica $\log$ abaixo ${ }^{(8,10,24)}$.

O músculo constritor inferior da faringe é constituído pelos dois fascículos musculares que se dirigem obliquamente de cima para baixo e de posterior para antero-lateral, inserindo-se a cada lado nas bordas laterais da cartilagem cricóide e pelo fascículo transverso, ímpar, com pouco mais de $1 \mathrm{~cm}$ de altura, cruzando a linha média, nas bordas póstero-laterais de um a outro lado, também na cartilagem cricóide e ainda os fascículos do tireofaríngeo. A porção tireofaríngea é mais espessa que os fascículos laminares do músculo cricofaríngeo e está situada logo acima, de modo a constituir o músculo constritor inferior da faringe (8,10-11,28). $^{\text {. }}$

Desta forma, o fascículo tireofaríngeo, acima da musculatura circular esofágica, participa da função esfinctérica em associação com o músculo cricofaríngeo, já que a zona de alta pressão excede em extensão o limite anatômico do cricofaríngeo $^{(8,10,24)}$.

A disfunção cricofaríngea é o distúrbio na deglutição localizado no esfíncter esofágico superior (EES). Tal distúrbio pode ocorrer em virtude das seguintes razões: uma doença no nervo central ou periférico, uma doença muscular, cirurgia prévia de pescoço ou idiopatia. A acalasia do cricofaríngeo é um distúrbio de motilidade em que o EES não se relaxa durante a deglutição, ocorrendo disfagia intermitente e aspiração ${ }^{(8,10)}$.

Muitas doenças, principalmente as neurológicas, revelam disfagia causada por distúrbios que afetam a função faríngea. Em pacientes com tais doenças, a disfunção do cricofaríngeo é um dos achados mais freqüentes ${ }^{(8,11,24)}$.

A terapêutica pela intervenção cirúrgica de miotomia do cricofaríngeo almeja contribuir para a melhora da dinâmica da deglutição, permeando a obstrução causada pela não abertura da TFE, por acesso externo ou endoscópico ${ }^{(8,11-12)}$.

Outro procedimento vem sendo discutido por ser menos invasivo: a aplicação de toxina botulínica no EES, buscando ter o mesmo efeito da miotomia do cricofaríngeo, ou seja, favorecer a passagem do bolo pela transição faringo esofágica $^{(29,30)}$.

A Toxina Botulínica (BtxA) é uma neurotoxina sintetizada do bacilo Clostidium botulinum, que age nas terminações nervosas colinérgicas pré-sinápticas, impedindo a libertação da acetilcolina na junção mioneuronal. Na prática, ela enfraquece a contração quando injetada no alvo do músculo ${ }^{(29)}$.

A Toxina Botulínica tipo A (Botulim toxin type A - BtxA) foi introduzida para pacientes com espasticidade, hipertonia ou demora na diminuição da tensão do esfíncter esofágico superior. Ocorrem melhoras, mas é necessária a re-injeção em 3-5 meses ${ }^{(11,22,29-32)}$. O procedimento ainda não se verifica como alternativa da miotomia. Além disso, várias complicações são relatadas.

A Toxina Botulínica tipo A representa valor terapêutico significativo no gerenciamento de uma variedade de distúrbios. Exemplos: blefaroespasmo, torticollis, distonia orofacial, disfonia espamódica, no tratamento de disfunções no nervo facial. Também no tratamento de alterações na motilidade esofageana, tais como: a acalasia, a hipertensão no esfíncter esofágico, inferior e o espasmo no esfíncter esofágico superior ${ }^{(11,22)}$.
Contudo, é importante salientar que são necessários estudos adicionais antes de oferecer fortes aprovações do procedimento em relação ao EES. Os pacientes que obtiveram melhores resultados pós-tratamento deveriam gozar de uma duração efetiva mais longa ${ }^{(31)}$, já que é necessária a re-injeção entre três e cinco meses. Assim, ainda não se verifica como alternativa da miotomia, objeto de estudo deste trabalho.

A miotomia do cricofaríngeo tem sido empregada há muito tempo, como parte do tratamento de disfagias de origem neurológica, miogênica, estrutural ou idiopática, mas sua eficácia permanece controversa. Além disso, indicações para o tipo de tratamento citado acima não são totalmente precisas $^{(5,12,31)}$, já que a consideração da função esfinctérica e da alta pressão regional não se devem ao estado de contração mantida do músculo cricofaríngeo, em decorrência das suas características anatômicas e por ser de tipo estriado esquelético ${ }^{(5,8)}$.

Para garantir a eficácia da miotomia (secção) do cricofaríngeo, é muito importante que a dinâmica hiolaríngea esteja preservada, com a elevação e anteriorização do complexo hiolaríngeo, a fim de que o pinçamento se desfaça pelo afastamento entre a coluna e a laringe, e a pressão intraluminal caia a nível zero ${ }^{(5,8)}$.

Associado a esta dinâmica é de igual importância um certo grau de pressurização faríngea e ejeção oral para que o conteúdo faríngeo seja conduzido através da $\mathrm{TFE}^{(5,8)}$.

Assim, provocando a abertura da TFE, são necessárias interação e sincronismo entre a força de ejeção oral e a dinâmica de elevação faríngea e laríngea. Tal ação interfere na qualidade da deglutição e na dinâmica de proteção laríngea ${ }^{(9)}$.

Somente a secção do fascículo transverso do cricofaríngeo deve ser processada para diminuir a resistência da TFE, pois a região acima deste ponto não oferece grande resistência, estando mais relacionada à pinça laringocervical. Se processada esta secção, a força ejetora desta musculatura ficará prejudicada $^{(8-10)}$.

A participação do cricofaríngeo na fisiopatologia das disfunções da TFE, por ser músculo estriado esquelético, pode gerar:

- espasticidade, nas doenças neurológicas (por exemplo: Acidente Vascular Encefálico, AVE, com comprometimento do sistema piramidal);

- rigidez, nas doenças neurológicas de acometimento extrapiramidal e

- nas doenças comuns, "disfunções primárias do cricofaríngeo", hipertonia ou hipertrofia ${ }^{(8,10,31)}$.

Em tais casos, é possível obter algum resultado positivo com a secção do fascículo transverso do cricofaríngeo, se houver pressurização faríngea (força de ejeção), mais a dinâmica de elevação e a anteriorização hiolaríngea nesta região ${ }^{(8-10)}$.

\section{DISCUSSÃO}

Devido a hiperatividade do esfíncter esofágico superior, mais especificamente do músculo cricofaríngeo, a disfagia ocorre em muitas doenças, por exemplo: acidente vascular encefálico, esclerose lateral amiotrófica, esclerose múltipla, neurinoma acústico e doença de Parkinson. Isto também pode 
acontecer depois de procedimentos cirúrgicos de cabeça e pescoço, como: ressecções da orofaringe e carcinoma supraglótico ${ }^{(31)}$.

Tradicionalmente, a miotomia cirúrgica do músculo cricofaríngeo é o tratamento de escolha para hiperatividade do esfíncter esofágico superior. Contudo, é preciso ressaltar, a miotomia do cricofaríngeo é um método invasivo e, segundo alguns autores, nem sempre efetivo ${ }^{(11,31-32)}$.

Por outro lado, a eficácia da aplicação da toxina botulínica para melhora da deglutição por alterações na transição faringo esofágica é limitada pela possibilidade de persistentes estenoses estruturais do EES e o risco de difusão de BtxA dentro da laringe ou hipofaringe ${ }^{(33)}$. Ainda assim, permanece a preferência pela miotomia do cricofaríngeo para os distúrbios nessa região.

As decisões clínicas - a partir da história do paciente, exame físico e os exames de manometria e videofluoroscopia - determinam a disfunção no EES e quais os melhores métodos para identificar os pacientes que devem se beneficiar da miotomia do cricofaríngeo ${ }^{(5-7)}$.

Quanto aos exames, a eletromiografia faríngea não é amplamente utilizada, mas poderia ser útil no estudo da deglutição normal, o que ajudaria a determinar precisamente o distúrbio envolvido e se haveria possibilidade de benefício com a miotomia ${ }^{(34)}$. A eletromiografia faríngea poderia auxiliar, portanto, uma vez que é necessário determinar o grau de constrição, hipertonia da região e se este é o fator principal do distúrbio. Mesmo assim, os exames de eleição para o estudo da fisiopatologia da deglutição continuam sendo a vídeofluoroscopia e a manometria, eventualmente laringoscopia e endoscopia digestiva alta ${ }^{(2)}$. Por tal razão, um dos indicadores principais para a adequada indicação de miotomia do cricofaríngeo seria a realização de avaliações instrumentais específicas, capazes de visualizar a dinâmica da deglutição, de modo a estudar a coordenação entre as fases oral, faríngea e esofágica (videofluoroscopia) e a mensuração dos valores pressóricos envolvidos nessa região (manometria).

Diferentes consistências e viscosidades dos alimentos também devem ser avaliadas para que se estude adequadamente o impacto dessas diferenças, na abertura da transição faringo esofágica, pois isto pode facilitar na terapia de reabilitação ${ }^{(20-21)}$.

Já o tratamento da disfunção orofaríngea é dependente da etiologia. Consiste em modificações da dieta, uso de técnicas de exercícios e manobras em deglutição, sondas nasoenterais e, em alguns casos, a realização de procedimentos cirúrgicos como a miotomia cricofaríngea ${ }^{(6)}$.

Outro aspecto fisiopatológico a ser destacado é a possível falta de coordenação, no relaxamento do esfíncter esofágico superior e inferior. Este fenômeno pode produzir o aumento da pressão intraluminal e protrusão da mucosa, com formação de divertículos que se encontram entre as fibras oblíquas do músculo constritor inferior da faringe, por cima do cricofaríngeo. A formação deste divertículo faringoesofágico, o de Zenker, se associa a transtornos de motilidade, tais como: acalasia, espasmo difuso e incremento da pressão intraluminal (por exemplo, a hérnia hiatal) ${ }^{(14)}$. Não há trata- mento fonoaudiológico para esse distúrbio, a ação deve ser cirúrgica (diverticulectomia e miotomia ou fundoplicatura e miotomia tipo Heller), realizando-se esofagograma, endoscopia e manometria ${ }^{(14-17)}$.

A disfagia secundária de acalasia não é freqüente em pacientes pediátricos; há, inclusive, o relato de dois casos de recorrentes pneumonias e aspiração que foram submetidas à miotomia com sucesso, confirmando a sua eficácia ${ }^{(34)}$.

A disfunção cricofaríngea primária é um distúrbio raro, idiopático e funcional do esfíncter esofágico superior, caracterizado por: disfagia, aspiração freqüente e um estreitamento funcional no nível do esfíncter esofágico superior ${ }^{(35)}$.

Melhoras depois da miotomia podem ser esperadas se os seguintes critérios forem cumpridos e observados na natureza da lesão neurológica:

- deglutição normal voluntária, ou seja, fase oral da deglutição preservada;

- movimento adequado de língua;

- função laríngea intacta e fonação;

- ausência de disartria ${ }^{(18)}$.

Uma pobre propulsão de língua pode atrasar o reflexo de início da deglutição e reduzir a peristalse faríngea e o não relaxamento do EES $^{(18)}$. Assim, a miotomia cricofaríngea poderia melhorar a disfagia orofaríngea neurogênica em pacientes com deglutição intacta na fase oral ${ }^{(18,19)}$.

A miotomia do cricofaríngeo pode aliviar a obstrução funcional resultante da incoordenação e espasticidade do EES. Isto é visto radiologicamente, quando observações préoperatórias e pós-operatórias são comparadas. A diminuição correspondente ao descanso e fechamento na pressão da junção faringoesofágica sugere que a diminuição na resistência do trânsito faringoesofágico é o mecanismo possível para explicar a resposta clínica ${ }^{(19)}$.

Mesmo aliviando a obstrução, o que se verifica é que, se o paciente não conseguir força e coordenação eficientes e suficientes para enviar o bolo coordenadamente para a câmara faríngea, ele não conseguirá uma deglutição segura ou eficiente, não ajudando, portanto, a indicação de miotomia para o retorno da via oral de alimentação ou mesmo a deglutição de saliva. Tal ocorrência faz com que se investigue qualquer distúrbio motor na cavidade oral, inclusive como mencionado anteriormente, a fala, nos casos de disartria.

Na manometria, observa-se que a contração na pressão do pico faríngeo não é afetada pela miotomia, pois tanto o repouso do EES como a pressão de contração, estão diminuídos pela operação. Já a incoordenação entre contração faríngea e descanso no EES é mais freqüentemente observa$\mathrm{da}$, nos pacientes com doenças neurológicas. A miotomia cricofaríngea não modifica esta disfunção. Prova disso é que em estudo pré e pós-operatório a estase hipofaríngea de resíduo persistiu depois da miotomia ${ }^{(18)}$.

Nos pacientes com elevação intacta da laringe a miotomia cricofaríngea pode aumentar a abertura do $\operatorname{EES}^{(19)}$. Uma vez que a pressão de repouso no EES não é função do cricofaríngeo, acredita-se que o cricofaríngeo está normalmente em estado de contração tônica, relaxando somente no instante crucial da deglutição ${ }^{(8,10,24)}$. Contudo, é importante destacar que estudos eletromiográficos em animais e huma- 
nos não detectaram qualquer contração tônica. Ao contrário, a contração do cricofaríngeo (em resposta a tal estímulo) se dá como distensão intraluminal, estimulação do nervo laríngeo superior e deslocamento da laringe e do osso hióide $^{(19)}$.

Assim, no estado de descanso, o EES está fechado por forças passivas nos tecidos arredores, ou seja, não há contração ativa do cricofaríngeo. Por isso, a miotomia do cricofaríngeo não beneficia pacientes que apresentam a elevação laríngea ausente durante a deglutição ${ }^{(19)}$.

A miotomia do cricofaríngeo não é efetiva em todas as ocorrências de disfagia. Ela deve ser específica para o tratamento em casos de disfagia causados por disfunção primária no músculo cricofaríngeo, podendo então falhar para aliviar disfagia em disfunções não idiopáticas, quando a fraqueza da propulsão faríngea está associada a disfunções do $\mathrm{EES}^{(22)}$.

A disfunção cricofaríngea é um distúrbio que pode ser causado por uma incoordenada deglutição faríngea, acalasia, ou a combinação desses fatores, sendo que esta falta de repouso do músculo cricofaríngeo pode ser documentada, antes do desenvolvimento do divertículo por estudos diagnósticos, o que permite ao cirurgião realizar a miotomia, aliviando os sintomas do paciente e prevenindo as seqüelas da disfunção do cricofaríngeo ${ }^{(7)}$.

Os sintomas incluem sensação de engasgo de um a dois segundos após a deglutição, dificuldades com sólidos e líquidos, rouquidão, tosses recorrentes, aspiração, regurgitação nasal e episódios recorrentes de pneumonia ${ }^{(7)}$.

Os resultados das pesquisas mostraram que a miotomia cricofaríngea deveria ser reservada para pacientes com distúrbio motor, limitado à fase faríngea da deglutição, falta de propulsão faríngea ou incoordenação cricofaríngea e/ou relaxamento incompleto ${ }^{(36)}$. Exceções para esta regra poderiam ser:

1) Diverticulum de Zenker, pois uma anormalidade pode não ser sempre detectada, mas os resultados da cirurgia demonstram a eficácia deste procedimento e,

2) queixas faringoesofágicas associadas ao refluxo - sendo que a maioria dos casos se resolve com a restauração da competência distal do esfíncter esofágico.

Para pacientes que apresentam múltiplos procedimentos anti-refluxo, a cautela deve ser usada na aplicação da miotomia, pois pode, inclusive, facilitar o retorno do conteúdo gástrico, uma vez que propicia maior abertura do $\mathrm{EES}^{(36)}$. Já os pacientes diagnosticados com hipertonia de esfíncter esofágico inferior têm apresentado melhora da disfagia ${ }^{(13)}$.

A primeira anormalidade cricofaríngea que resulta no divertículo de Zenker tem sido recentemente mostrada pela incompleta abertura do EES, enquanto a coordenação neuromuscular faringoesfágica e o relaxamento do esfíncter são normais ${ }^{(37)}$. A abertura inadequada do EES aumenta consideravelmente a pressão intrabolus hipofaríngea durante o fluxo do bolo trans-esfinctérico, o qual pode causar a bolsa de herniação ${ }^{(37)}$.

A provável causa da abertura inadequada do esfíncter é a degeneração das fibras musculares. Este achado sugere que o divertículo de Zenker resulte na diminuição da eficiência da função do $\operatorname{EES}^{(37)}$. A miotomia cricofaríngea realizada em pacientes com este tipo de achado fisiopatológico da deglutição, não resultou em alteração no tempo de início do relaxamento do EES durante a deglutição. A normalização da pressão intrabolus hipofaríngea, causou uma redução marginal na pressão basal de $\operatorname{EES}^{(37)}$, resultando em uma abertura eficiente.

Por isso, a miotomia cricofaríngea obtém bons resultados para divertículo de Zenker ${ }^{(37)}$, o que confirma a sua eficácia, pois ficou comprovada a oportunidade de estudar a relação entre a abertura do EES e a pressão intrabolus. Esses parâmetros, talvez em combinação, podem ser valiosos prognósticos de resultado, depois da miotomia cricofaríngea para disfagia de outras causas ${ }^{(37)}$.

Muitos pacientes com esclerose lateral amiotrófica obtêm melhora da disfagia com a miotomia cricofaríngea, a qual é feita na tentativa de aliviar a angústia de engasgar-se com comida e secreções, reduzindo o risco de pneumonia por aspiração e má nutrição ${ }^{(38)}$. Deve-se considerar o caráter progressivo da esclerose lateral amiotrófica para, somente após a compreensão do diagnóstico, definir condutas terapêuticas reabilitadoras da deglutição. Quem sabe, até, discutir a possibilidade de vias alternativas de alimentação, pois em razão das alterações neuromotoras, como a fraqueza e a atrofia muscular - o paciente pode apresentar dificuldades em todos os estágios e fases da deglutição, tais como: levar o alimento à boca, realizar a ejeção oral, controlar a secreção oral (o que gera acúmulo de resíduos na cavidade oral, recessos, valécula), fraqueza mastigatória e, também, dificuldade em realizar uma higiene oral adequada ${ }^{(3)}$.

Assim, o raciocínio clínico deve considerar a progressão da doença, evitando desnutrição, desidratação e complicações pulmonares, sendo mais bem conduzido por uma equipe coordenada e interdisciplinar de especialistas que decidirão se a miotomia será efetiva em determinado estágio da doença neuromuscular.

Nas disfagias paralíticas, alguns músculos não trabalham na extensão normal, sendo difícil enviar o bolo para o esôfago ${ }^{(39)}$. Além disso, por causa da possível incompetência nasofaríngea ou laríngea, podem ocorrer regurgitação nasal ou aspiração ${ }^{(39)}$. Esses pacientes não são capazes de realizar uma vigorosa pressão intrafaríngea, pois a duração da inibição da ação da válvula feita pelo músculo cricofaríngeo não é suficientemente longa para o bolo ser direcionado para o esôfago, sendo a duração menor que $0,5 \operatorname{segundos}^{(39)}$.

Recentes estudos têm revelado a complexidade da fase orofaríngea da deglutição e enfatizado a importância da abertura cricofaríngea em coordenação com a transferência do bolo através do EES. A maior contribuição da abertura é proporcionada pelo controle e força de ejeção oral eficientes e pelo movimento de anteriorização, elevação e estabilização do conjunto hiolaríngeo ${ }^{(5,8)}$. A completa distensão do esfíncter é modulada pelas pressões intrabolo que são, em turnos, geradas primariamente pela ação de pistão da língua, antes da peristalse faríngea ${ }^{(9)}$. Assim, considerando a importância da fase oral, a ação de pistão da língua, na verdade determina a peristalse faríngea, por isso a importância da coordenação entre as fases oral e faríngea da deglutição. 


\section{COMENTÁRIOS FINAIS}

O objetivo deste estudo foi verificar os critérios de indicação e eficácia da miotomia do cricofaríngeo nos pacientes com disfagia orofaríngea. Para isto foi realizada uma revisão da literatura sobre este procedimento e a descrição de seus resultados na dinâmica da deglutição.

Conclui-se que:

- é necessária a realização de exames como a videofluoroscopia e a manometria, a fim de determinar precisamente o fenômeno do distúrbio da deglutição para essa indicação;

- O paciente não deve ter distúrbios motores de fase oral, dificuldade de elevação laríngea e fraqueza ou incoordenação faríngea, pois, nestes casos, mesmo com maior abertura da transição faringo esofágica, o transporte do bolo continuará comprometido.

A baixa morbidade e mortalidade associadas ao procedimento têm encorajado a indicação de miotomias do cricofaríngeo para melhora da deglutição em pacientes com disfagia neurológica ou acalasia de cricofaríngeo. Porém, como este trabalho procura demonstrar, a indicação do procedimento deve ser feita considerando os antecedentes e evolução da doença - basicamente pelo estudo da fisiologia da deglutição.

\section{AGRADECIMENTOS}

Agradecemos ao CEFAC - Centro de Estudos em Fonoaudiologia Clínica, pelo incentivo e oportunidade de desenvolver esta pesquisa.

\begin{abstract}
Cricopharyngeal myotomy is a surgical procedure that might be indicated to patients with oropharyngeal dysphagia that, in general, are characterized by a motor impairment limited to the pharyngeal phase of swallowing, cricopharyngeal incoordination or incomplete relaxation of the Upper Esophageal Sphincter (UES). Indication of the myotomy, however, have been discussed and applied in many cases, with no effect on the improvement of deglutition dynamics. The aim of this study was to verify the criteria of indication and efficacy of cricopharingeal myotomy, based on studies of deglutition physiology in patients with oropharyngeal dysphagia. A literature review about this procedure and the description of its results on deglutition dynamics was carried out. Results showed that the best indicators of a well-succeeded myotomy are: efficient oral phase and good laryngeal elevation during deglutition. Therefore, an adequate indication of cricopharyngeal myotomy must consider not only the pharyngeal phase and the isolated functioning of the UES, but also the coordination and the relationship between the oral and the pharyngeal phases of deglutition.
\end{abstract}

KEYWORDS: Pharyngeal muscles/surgery; Cricoid cartilage/surgery; Esophageal sphincter, upper/physiology; Pharyngeal muscles/ physiology

\title{
REFERÊNCIAS
}

1. Souza BBA, Martins C, Campos DJ, Balsini IA, Meyer LR. Nutrição \& Disfagia: guia para profissionais. Curitiba: NutroClínica; 2003. p.67.

2. Jaradeh SS, Shaker R, Toohill RB. Eletromyographic recording of the cricopharyngeus muscle in humans. Am J Med. 2000;108 Suppl 4a:40S42S. Review.

3. Hernandez AM, Marchesan IQ. Atuação fonoaudiológica no ambiente hospitalar. Rio de Janeiro: Revinter; c2001. p.8,10-11.

4. Olsson R, Kjellin O, Ekberg O. Videomanometric aspects of pharyngeal constrictor activity. Dysphagia. 1996;11(2):83-6.

5. Kelly JH. Management of upper esophageal sphincter disorders: indications and complications of myotomy. Am J Med. 2000;108 Suppl $4 \mathrm{a}: 43 \mathrm{~S}-46 \mathrm{~S}$

6. Quera P R, Defilippi Caffri C. Disfagia orofaríngea. Gastroenterol Latinoam. 2001;12(1):26-35.

7. Halvorson DJ, Kuhn FA. Transmucosal cricopharyngeal myotomy with the potassium - titanyl - phosphate laser in the treatment of cricopharyngeal dysmotility. Ann Otol Rhinol Laryngol. 1994;103(3):173-7.

8. Costa MMB, Castro LP. Tópicos em deglutição e disfagia. Rio de Janeiro: Medsi; 2003. p.387-93.

9. Costa MMB, Moscovici M, Pereira AA, Koch HA. Avaliação videofluoroscópica da transição faringoesofágica (esfíncter superior do esôfago). Radiol Bras. 1993;26(2):71-80.
10. Costa MMB. Análise estrutural da laringofaringe e suas implicações na miotomia do cricofaríngeo, na injeção de toxina botulínica e na dilatação por balão. Arq Gastroenterol. 2003;40(2):63-72.

11. Haapaniemi JJ, Laurikainem EA, Pulkkinen J, Marttila RJ. Botulinum toxin in the treatment of cricopharyngeal dysphagia. Dysphagia. 2001;16(3):171-5.

12. Ali GN, Wallace KL, Laundl TM, Hunt DR, deCarle DJ, Cook IJ. Predictors of outcome following cricopharyngeal disruption for pharyngeal dysphagia. Dysphagia. 1997;12(3):133-9.

13. Pereira-Lima JC, Lopes CV, Cirne RVM, Coelho NHV. Toxina botulínica no tratamento da acalasia: relato de três casos e revisão da literatura. Rev AMRIGS. 1998;42(3):168-71.

14. Muraro CPM, Aquino JLB, Silva MRM, Lintz JE. Divertículo de Zenker: análise de 11 pacientes. Rev Ciências Médicas. 1997;6(2/3):958 .

15. Sánchez Fernández P, Castillo González A, Mier y Díaz J, Robledo Ogazón F, Blanco Benavides R. Experiencia en el manejo de divertículos esofágicos. Cir \& Cir. 1999;67(2):54-8.

16. de la Garza Villaseñor L, Cortés Gonzáles R. Tratamiento quirúrgico del divertículo faringoesofágico de Zenker. Rev Gastroenterol Méx. 1996;61(4):320-6.

17. Saad E, Serrano L, Lafuente A, Hansen M, Kido N. Divertículo de Zenker: nuestra experiencia. Prensa Méd Argent. 1997;84(3):204-6. 
18. Poirier NC, Bonavina L, Taillefer R, Nosadini A, Peracchia A, Duranceau A. Cricopharyngeal myotomy for neurogenic oropharyngeal dysphagia. J Thorac Cardiovasc Surg. 1997;113(2):233-40; discussion 240-1.

19. Woodson G. Cricopharyngeal myotomy and arytenoid adduction in the management of combined laryngeal and pharyngeal paralysis. Otolaryngol Head Neck Surg. 1997;116(3):339-43.

20. Ren J, Shaker R, Zamir Z, Dodds WJ, Hogan WJ, Hoffmann RG. Effect of age and bolus variables on the coordination of the glottis and upper esophageal sphincter during swallowing. Am J Gastroenterol. 1993;88(5):665-9.

21. Dantas RO, Dodds WJ. Influência da viscosidade do bolo alimentar deglutinado na motilidade da faringe. Arq Gastroenterol. 1990;27(4):164-8.

22. Lacau St Guily J, Zhang KX, Périé S, Copin H, Butler-Browne GS, Barbet JP. Improvement of dysphagia following cricopharyngeal myotomy in a group of elderly patients. Histochemical and biochemical assessment of the cricopharyngeal muscle. Ann Otol Rhinol Laryngol. 1995;104(8):603-9.

23. Macedo Filho ED, Gomes GF, Furkim AM. Manual de cuidados do paciente com disfagia. São Paulo: Lovise; 2000. p. 23-6,112.

24. Silvarao DV, Goyal RK. Functional anatomy and physiology of the upper esophageal sphincter. Am J Med. 2000;108 Suppl 4a:27S-37S

25. Holloway RH. Esophageal body motor response to reflux events: secondary peristalsis. Am J Med. 2000;108 Suppl 4a:20S-26S.

26. Hornby PJ, Abrahams TP. Central control of lower esophageal sphincter relaxation. Am J Med. 2000;108 Suppl 4a:90S-98S.

27. Yamaoka M, Furusawa K. Function of the inferior pharyngeal constrictor muscle. Dysphagia. 1997;12(3):171.

28. Alipour F, Titze I. Active and passive characteristics of the canine cricothyroid muscles. J Voice. 1999;13(1):1-10.
29. Shaw GY, Searl JP. Botulinum toxin treatment for cricopharyngeal dysfunction. Dysphagia. 2001;16(3):161-7.

30. Macedo Filho ED. Uso da toxina botulínica no tratamento da disfagia orofaríngea. In: Costa MMB, Castro LP. Tópicos em deglutição e disfagia. Rio de Janeiro: Medsi; 2003. p. 395-405.

31. Chiu MJ, Chang YC, Hsiao TY. Prolonged effect of botulinum toxin injection in the treatment of cricopharyngeal dysphagia: case report and literature review. Dysphagia. 2004;19(1):52-7.

32. Moerman M, Callier Y, Dick C, Vermeersch H. Botulinum toxin for dysphagia due to cricopharingeal dysfunction. Eur Arch Otorhinolayngol. 2002;259(1):1-3.

33. Alberty J, Oelerich M, Ludwig K, Hartmann S, Stoll W. Efficacy of botulinum toxin A for treatment of upper esophageal sphincter dysfunction. Laryngoscope. 2000;110(7):1151-6.

34. Mahomed AA. Primary cricopharyngeal achalasia in infancy myotomy treatment of choice. S Afr J Surg. 2000;38(2):28-30.

35. Solt J, Bajor J, Moizs M, Grexa E, Horváth PO. Primary cricopharyngeal dysfunction: treatment with balloon catheter dilatation. Gastrointest Endosc. 2001;54(6):767-71.

36. Bonavina L, Khan NA, DeMeester TR. Pharyngoesophageal dysfunctions. The role of cricopharyngeal myotomy. Arch Surg. 1985;120(5):541-9.

37. Shaw DW, Cook IJ, Jamieson GG, Gabb M, Simula ME, Dent J. Influence of surgery on deglutitive upper oesophageal sphincter mechanics in Zenker's diverticulum. Gut. 1996;38(6):806-11.

38. Lebo CP, U KS, Norris FH Jr. Cricopharyngeal myotomy in amyotrophic lateral sclerosis. Laryngoscope. 1976;86(6):862-8.

39. Hirano M. Cricopharyngeal myotomy for paralytic dysphagia. JFORL J Fr Otorhinolaryngol Audiophonol Chir Maxillofac. 1974;23(8):7324 . 